\title{
Asporin participates in gastric cancer cell growth and migration by influencing EGF receptor signaling
}

\author{
QIAN DING, MEI ZHANG and CAN LIU \\ Department of Ultrasound, The First Affiliated Hospital of Yangtze University, \\ Jingzhou, Hubei 434000, P.R. China
}

Received October 19, 2014; Accepted January 16, 2015

DOI: 10.3892/or.2015.3791

\begin{abstract}
Asporin (ASPN), a novel member of the small leucine-rich proteoglycan (SLRP) family, serves as a key component of the tumor stroma and has been reported to be abnormally expressed in certain types of tumors. Specifically, the proteoglycan was proven to activate the coordinated invasion of scirrhous gastric cancer and cancer-associated fibroblasts. However, the role of ASPN in cancer cell growth and metastasis has not yet been addressed. In the present study, we aimed to evaluate the tumoricidal benefits of ASPN on tumorigenesis and progression of gastric cancer. Firstly, it was demonstrated that ASPN was overexpressed in gastric carcinoma tissues when compared to the corresponding non-cancerous tissues, and it had varied levels of expression in gastric cancer epithelial cell lines. Additionally, we assessed the effects of transient siRNA-mediated ASPN knockdown on gastric cancer cells. ASPN silencing inhibited proliferation and suppressed the migration of immortalized neoplastic epithelial cells. Furthermore, at the molecular level, we found that downregulation of ASPN blocked the anti-apoptotic molecule Bcl-2, increased the expression of pro-apoptotic molecule $\mathrm{Bad}$, reduced the expression of migration-related proteins CD44 and matrix metalloproteinase (MMP)-2, and abrogated the activation of the phosphorylation status of ERK and epidermal growth factor (EGF) and its receptor (EGFR). Collectively, our findings indicate that ASPN is upregulated and plays an oncogenic role in gastric cancer progression and metastasis by influencing the EGFR signaling pathway.
\end{abstract}

\section{Introduction}

As one of the most common malignancies worldwide, gastric cancer is a complex disease and is frequently characterized by genetic and epigenetic alterations $(1,2)$. The interaction

Correspondence to: Dr Can Liu, Department of Ultrasound, The First Affiliated Hospital of Yangtze University, 8 HangKong Road, Jingzhou, Hubei 434000, P.R. China

E-mail: liucancj@sohu.com

Key words: asporin, gastric cancer, proliferation, migration, EGF receptor signaling of genetic predispositions with environmental factors is one major pathogenesis of this cancer (3). Metastasis, the most critical aspect of tumorigenesis, is considered as a leading cause of cancer-related mortality $(3,4)$. Thus, important goals of cancer research are the identification of key genes and signaling pathways involved in metastasis and tumor progression, which may facilitate the prediction of those events and hereby identify therapeutic targets.

It is generally accepted that the tumor environment determines the behavior of malignant cells. In the tumor environment, extracellular matrix (ECM) proteoglycans are essential components and represent a complex structural entity that supports and interacts with epithelial structures, playing crucial pathological roles (5). Asporin (ASPN), a novel member of the small leucine-rich repeat proteoglycan (SLRP) family, contains a unique aspartate-rich $\mathrm{N}$ terminus and is involved in the stromal modification of ECM (6). The expression levels of ASPN and other related matrix proteoglycans, for instance decorin and biglycan, have been assessed in gastric pathology, and are implicated in the oncogenesis and development of human gastric carcinoma (7-10). Particularly, Satoyoshi et al recently concluded that ASPN activates the coordinated invasion of scirrhous gastric cancer and cancer-associated fibroblasts by activation of the CD44-Racl pathway (7). In addition, by applying quantitative mass spectrometry, innovative proteomic technologies or microarray analysis, ASPN was proven to be upregulated in a series of cancers, including pancreatic cancer (11), pancreatic ductal adenocarcinoma (12), prostate cancer (13) and breast carcinoma (14). In osteoarthritis, Kizawa et al found a direct interaction between ASPN and TGF- $\beta$. An ASPN allele with a 14-aspartic acid repeat in the $\mathrm{N}$-terminal region, was designated as D14. The frequency of the D14 allele was found to increase with disease severity, and the effect of ASPN on TGF- $\beta$ activity was allele-specific (15). However, the exact roles of ASPN responsible for tumor cell proliferation and migration and the specific molecular mechanisms have not been fully elucidaded.

Epidermal growth factor (EGF) and its receptor (EGFR) are closely associated with tumorigenesis. Overexpression or activation of these has been observed in a variety of tumor types and cell lines, including gastric cancer $(16,17)$. Binding of EGF to EGFR may accelerate cell proliferation and migration, and trigger epithelial cell signaling in gastric cancer (18). The MAPK/ERK pathway is a classic downstream signaling 
pathway of EGFR signaling (19). The Bcl-2 gene family could be regulated by the MAPK/ERK pathway. The homeostatic balance between pro-apoptotic and anti-apoptotic homodimers (such as Bax and Bcl-2) can reflect cellular apoptosis or proliferation (20). CD44, a cell surface molecule, is associated with EGFR and is involved in diverse cell-cell and cellmatrix interactions, resulting in the metastasis of mammary carcinoma cells (21). In addition, matrix metalloproteinases (MMPs), particularly the gelatinase subfamily MMP-2, has been considered to participate in tumor progression and metastasis in many types of cancers (22).

Therefore, based on these previous findings, the present study aimed to further investigate the expression and the specific cellular function of ASPN in human primary gastric adenocarcinomas, to extend the knowledge of this matrix proteoglycan in the development and progression of cancer.

\section{Materials and methods}

Clinical materials. Pairs of primary gastric carcinoma tissues and their corresponding non-neoplastic gastric mucosal tissues $(\geq 5 \mathrm{~cm}$ ) were acquired from 46 gastric cancer patients who underwent curative surgery at the First Affiliated Hospital of Yangtze University. Written informed consent was obtained from all the participants according to a protocol approved by the Yangtze University of Medicine Institutional Review Board. The characteristics of the patients were obtained from medical records and are summarized in Table I. Portions of the tissues were processed for immunohistochemistry, quantitative real-time PCR and western blot analyses. All samples were frozen immediately in liquid nitrogen and stored at $-80^{\circ} \mathrm{C}$ until required.

Immunohistochemical analysis. Gastric tissues were formaldehyde-fixed and paraffin-embedded. Sections $(5-\mu \mathrm{m})$ were deparaffinized in Citrisolve and rehydrated through graded ethanol. Immunohistochemical staining was performed using an EnVision kit (Dako, Carpinteria, CA, USA) according to the manufacturer's protocol. The sections were incubated with a primary antibody against ASPN (Sigma-Aldrich, St. Louis, MO, USA) or the negative control (normal rabbit IgG; Cell Signaling Technology, Danvers, MA, USA). Analysis of the immunohistochemical staining was performed independently by two pathologists. Five random fields from each section were photographed and analyzed with Image-Pro Plus 6.0 software.

Cell lines and culture. The human normal gastric epithelial cell line GES and, human gastric cancer cell lines SGC-7901 and BGC-823 were purchased from the Institute of Biochemistry and Cell Biology of the Chinese Academy of Science. Human gastric cancer cell lines AGS, MKN45 and N87 were obtained from the China Center for Type Culture Collection. All of the cell lines were maintained in RPMI-1640 (Hyclone, Logan, UT, USA) medium, and the media were supplemented with $10 \%$ FBS (Hyclone), $100 \mathrm{U} / \mathrm{ml}$ penicillin, $100 \mu \mathrm{g} / \mathrm{ml}$ streptomycin sulphate and $1 \mu \mathrm{M}$ sodium pyruvate. The cells were cultured at $37^{\circ} \mathrm{C}$ in a humidified incubator with $5 \% \mathrm{CO}_{2}$. The cells were passaged with trypsin-EDTA $(0.05 \%$ trypsin and $0.53 \mu \mathrm{M}$ tetrasodium EDTA).
Table I. Clinical characteristics of the gastric cancer patients.

\begin{tabular}{lc}
\hline Characteristics & No. of patients $(\%)(\mathrm{N}=46)$ \\
\hline Gender & $27(58.70)$ \\
Male & $19(41.30)$ \\
Female & \\
Age (years) & $24(52.17)$ \\
$>60$ & $22(47.83)$ \\
$\leq 60$ & \\
Tumor size (cm) & $25(54.35)$ \\
$<6$ & $21(45.65)$ \\
$\geq 6$ & $17(36.96)$ \\
Type & $16(34.78)$ \\
Tubular adenocarcinoma & $13(28.26)$ \\
Mucosal adenocarcinoma & \\
Signet-ring cell carcinoma & $19(41.30)$ \\
Clinical stage & $27(58.70)$ \\
I and II & \\
III and IV & \\
\hline
\end{tabular}

Plasmid construction and cell transfection. The ASPN silencing plasmid shRNA was constructed by Shanghai GenePharma Co., Ltd. (Shanghai, China) targeting the gene aspn (Gene Bank accession no. NM_017680), and was cloned into the mammalian expression vector GV248 with AgeI and EcoRI restriction enzyme sites. Four siRNAs targeting the indicated genes were designed as follows: ASPN-RNAi (26538), CGG TGA AAT ACT GGG AAA T; ASPN-RNAi (26539), TCT TGA TAA TAA TGG GAT A; ASPN-RNAi (26540), CTC TTG ATA ATA ATG GGA T; and ASPN-RNAi (26541), AAC CAA CAT TCC ATT TGA T.

According to the manufacturer's specifications, human gastric cancer cells were transfected with siRNA-ASPN-GV248 or siRNA-NC-GV248 with Lipofectamine 2000 transfection reagent (Invitrogen, Gaithersburg, MD, USA) in 6-well plates. The plasmid expressing EGFP was used to evaluate the transfection efficacy.

Quantitative real-time PCR analyses. Total RNA from the cells or the frozen tissues was extracted with TRIzol reagent (Takara, Dalian, China) according to the manufacturer's instructions. The RNA quality (A260/A280 ratio) and quantity were determined using a standard spectrophotometer. One microgram of RNA was reversely transcribed using the RevertAid ${ }^{\mathrm{TM}}$ First Strand cDNA Synthesis kit (\#1622; Fermentas, Vilnius, Lithuania) at $42^{\circ} \mathrm{C}$ for $45 \mathrm{~min}$, followed by $70^{\circ} \mathrm{C}$ for $5 \mathrm{~min}$. Quantitative real-time PCR was performed using QPK-201 SYBR-Green Master Mix (Toyobo, Osaka, Japan) on a 7300 Real-time PCR system (Applied Biosystems, Foster City, CA, USA). The target cDNA-specific primers of ASPN were utilized as described previously (14). GAPDH was used as the normalization control, and duplicate experiments were performed for ASPN mRNA measurement. The mRNA expression level was determined using the $2^{(-\Delta \Delta C t)}$ method. 
Cell viability assay. AGS cells were seeded onto 96-well plates for $24 \mathrm{~h}$ after siRNA-ASPN-GV248 or siRNA-NC-GV248 transfection at a density of 3,000 cells/well (day 0). Cell viability was assessed on day 24, 48 and $72 \mathrm{~h}$, using the Cell Counting Kit-8 assay (CCK-8; Dojindo Laboratories, Tokyo, Japan). Ten microliters of reagent was added to each well, incubated at $37^{\circ} \mathrm{C}$ for $1 \mathrm{~h}$, and the absorbance was detected at $450 \mathrm{~nm}$ according to the manufacturer's instructions. With 5 replicates in each experiment, independent experiments were repeated 3 times.

Colony formation assay. AGS cells transfected with siRNA-ASPN-GV248 or the GV248 empty vector were selected with G418-resistance (Sigma-Aldrich) for 2 weeks. The stably transfected cells were cultured in 6-well plates (500/well) until colonies formed consisting of more than 50 cells. Then the cells were fixed in cold-methanol and stained with crystal violet solution. The colonies were counted with Quantity One software (Bio-Rad, Hercules, CA, USA) and photographed. The experiment was performed in triplicate wells in 3 independent experiments.

Transwell assay. Transwell assays were performed after the expression of ASPN was downregulated to observe the direct effect of the apsn gene on the migration of gastric cancer cells. Briefly, Transwell inserts with 6.5-mm diameter polycarbonate $8-\mu \mathrm{m}$ microporous membranes (Corning, Inc., Corning, NY, USA) were placed over the bottom chambers that were filled with culture medium containing $20 \%$ FBS. The AGS cells that transfected for $24 \mathrm{~h}$ with siRNA-ASPN-GV248 or siRNA-NC-GV248 were suspended in RPMI-1640 medium containing $1 \%$ FBS and added to the upper chamber. After another 20-h incubation, cells on the upper surface of the well were removed with a cotton swab, and the migrated cells were fixed in cold methanol and stained with crystal violet (23). The number of transmigrated cells in 5 random fields from each well was counted and photographed. Each experiment was performed in triplicate, and migration was expressed as the mean \pm SD of total cells counted per field.

Wound-healing assay. Wound-healing assays were performed as described previously (23). Briefly, after transfecting with siRNA-ASPN-GV248 or siRNA-NC-GV248 for $24 \mathrm{~h}$, the cells were seeded in 6 -well plates $\left(2.0 \times 10^{6}\right.$ cells/well). Once a confluent cell monolayer formed, it was scraped using a yellow sterile pipette tip to generate a scratch wound. Then, the cells were washed with PBS to remove the cellular debris. The migration of the cells into the scratch was observed and photographed at time-points of 0,10 and $20 \mathrm{~h}$. Wound areas were calculated to determine the migrated distance. Experiments were performed in triplicate.

Protein extraction and immunoblotting. Total protein was prepared from the human tissue specimens and treated cells with RIPA lysis buffer. The protein concentration was determined using the protein assay reagent (BCA; Beyotime, Shanghai, China). The protein extracts were boiled with $5 \mathrm{X}$ loading buffer. Equal amounts of protein $(40 \mu \mathrm{g})$ were separated on polyacrylamide gel and electro-transferred, transferred to a nitrocellulose membrane, and then blocked with $5 \%$ non-fat dry milk in TBS containing $0.1 \%$ Tween-20. The following primary antibodies were used for the assays: antiASPN (Sigma-Aldrich), anti-Bcl-2, anti-Bax, anti-ERK and anti-p-ERK (Cell Signaling Technology), anti-EGFR, anti-pEGFR and anti-CD44 (Peprotech, Inc., Rocky Hill, NJ, USA), anti-MMP-2 (Abcam, Cambridge, UK) and anti-GAPDH (Bioworld Technology Inc., St. Louis. Park, MN, USA). The blots were visualized with a HRP-conjugated antibody followed by chemiluminescence reagent (Millipore, Billerica, MA, USA) detection on photographic film. The target protein was normalized to GAPDH through a comparison of the gray scale values, and the analysis was performed with Quantity One software.

Statistical analysis. Quantitative data are presented as means \pm SD. GraphPad Prism version 5.0 for Windows was used for all statistical analysis, and the Student's t-test was used to compare the values of the test and control samples. $\mathrm{P}<0.05$ was considered to indicate a statistically significant result.

\section{Results}

Abnormally expressed ASPN in gastric cancer. To evaluate the expression of ASPN in gastric cancer, 46 pairs of primary gastric carcinoma tumors and matched adjacent non-tumor tissues were analyzed by immunohistochemical staining. The sections showed markedly increased ASPN staining (31/46) in cancer relative to the matching non-cancerous gastric mucosa samples from the same patients (Fig. 1A). Additionally, the aberrant ASPN expression was confirmed by western blot analysis in 10-paired gastric carcinoma tissues. This analysis also revealed that ASPN protein levels were significantly increased in the primary tumor tissues when compared to the tumor adjacent tissues (Fig. 1B and C). Furthermore, ASPN mRNA expression was investigated in gastric cancer with quantitative real-time PCR. There was an insignificant trend toward ASPN mRNA upregulation in the gastric cancer tissues relative to the matched adjacent non-tumor tissues (Fig. 1D). Taken together, these results suggest that ASPN is aberrantly expressed in gastric cancer and the proteoglycan may function as an oncogene in gastric carcinogenesis.

ASPN has varied expression in gastric cancer epithelial cell lines. We further examined ASPN protein levels in different gastric cancer epithelial cell lines, including AGS, MKN28, SGC-7901, N87, BGC-823, MKN45, and the human immortalized gastric mucosa epithelial cell GES. Among these cell lines, as shown in Fig. 1E, the poorly differentiated adenocarcinoma cell lines AGS, BGC-823 and MKN45 exhibited relatively high levels of ASPN expression; the moderately differentiated adenocarcinoma cell line SGC-7901 and the well-differentiated adenocarcinoma cell line N87 revealed low levels of ASPN; and the human immortalized gastric mucosa epithelial cell line GES showed apparently low levels of ASPN (2). Collectively, the results suggest that ASPN expression is varied in an inverse trend following the differentiation degree of gastric cancer epithelial cell lines, at least to some extent.

ASPN is required for the proliferation of gastric cancer cells. To understand the potential function of ASPN in gastric cancer, 


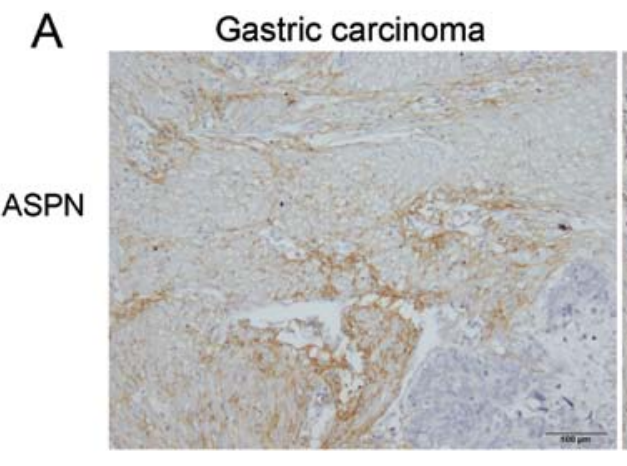

\section{Adjacent normal control}
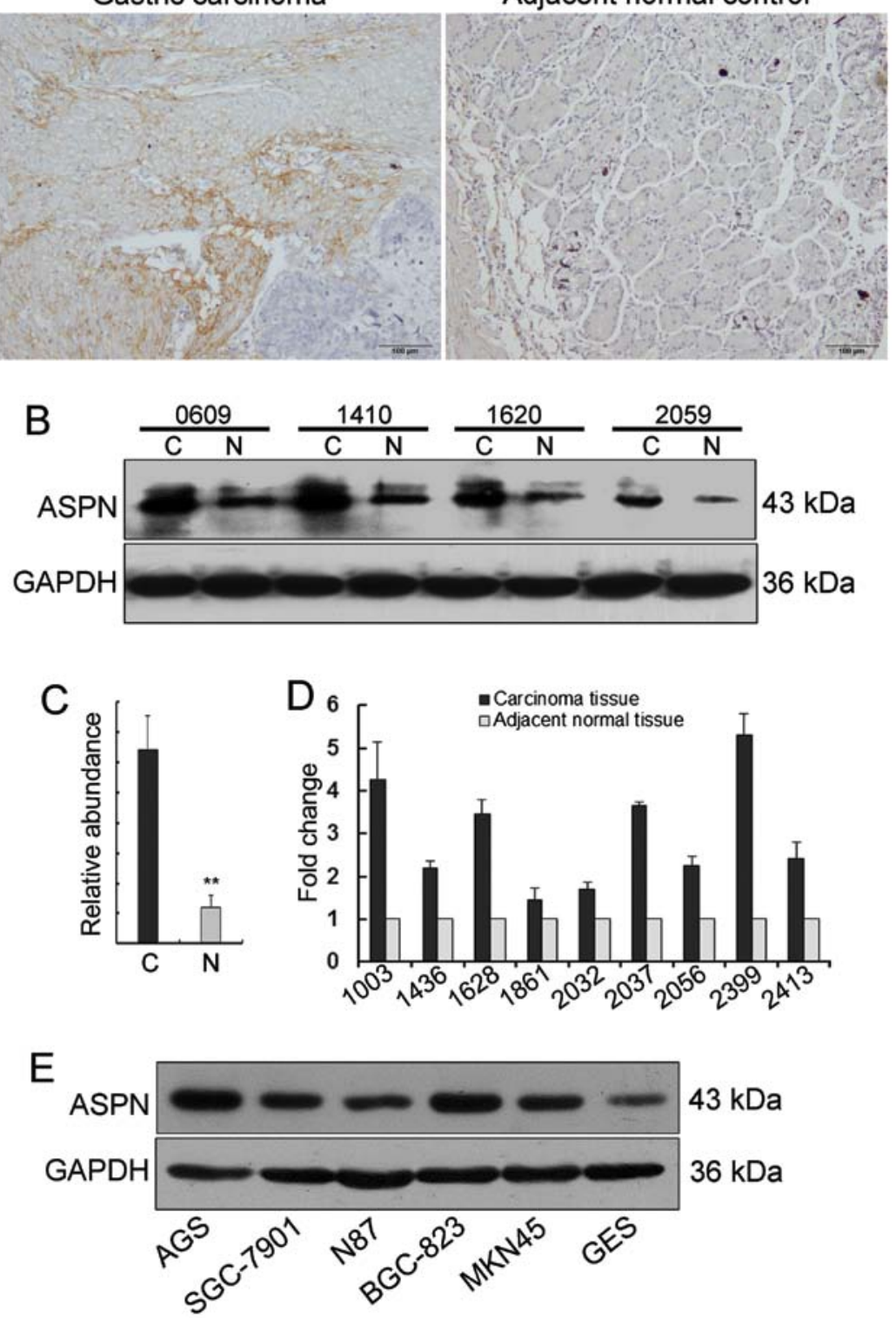

Figure 1. Identification of differential ASPN expression in gastric carcinoma tissues and cells. (A) Immunohistochemical staining of ASPN in human gastric carcinoma (left) and adjacent normal (right) specimens. (B) Expression of ASPN protein in gastric carcinoma tissues and the matched adjacent non-tumor tissues. C, carcinoma tissues, N, surrounding non-tumor tissues. (C) The intensity of ASPN bands of the western blot analysis in 10 pairs of gastric carcinoma tissues and corresponding normal tissues was quantified by scanning densitometry. ${ }^{* *} \mathrm{P}<0.01$. (D) Quantitative real-time PCR showed that ASPN expression was upregulated in gastric carcinoma tissues. (E) Western blotting revealed that ASPN had varied expression in different gastric cancer epithelial cell lines. ASPN, asporin.

the most highly ASPN-expressing AGS cells were transfected with siRNA-ASPN-GV248 or siRNA-NC-GV248 vector. The transfection efficiency was identified and confirmed by quantitative real-time PCR and western blotting. Among the 4 siRNAs targeting for the indicated gene, siRNA-ASPN (26540, \#40) induced the highest level of downregulation (Fig. 2A and B). The effect of ASPN on the cell proliferation in AGS cells was confirmed by CCK-8 assay, which showed significant reduction in cell viability in the siRNA-ASPN-transfected cells at the time-points of 48 and $72 \mathrm{~h}$, but not at $24 \mathrm{~h}$, compared with the control vector transfectants (Fig. 2C). In addition, similar results were revealed in the colony formation assay. The cells stably transfected with siRNA-ASPN displayed a markedly decreased number of surviving colonies forming on the plates (Fig. 2D). Collectively, these results indicate that aberrant expression of ASPN may be critical for the proliferation and survival of gastric tumor cells.

ASPN silencing suppresses the migration of gastric cancer cells. Increased cell motility is one of the properties shared by cancer cells. Thus, Transwell and wound-healing assays were performed to further determine whether the downregulation of ASPN influences the migratory ability of gastric cancer cells. The results of the Transwell assay indicated that the numbers of cells in the siRNA-ASPN-GV248 (\#40) group that migrated to the lower surfaces were significantly reduced at the time-points of $20 \mathrm{~h}$, in comparison to these numbers in the siRNA-NC and untreated control groups (Fig. 3A). Similarly, the wound-healing assay showed that ASPN silencing apparently induced less migrating cells than did the control siRNA 


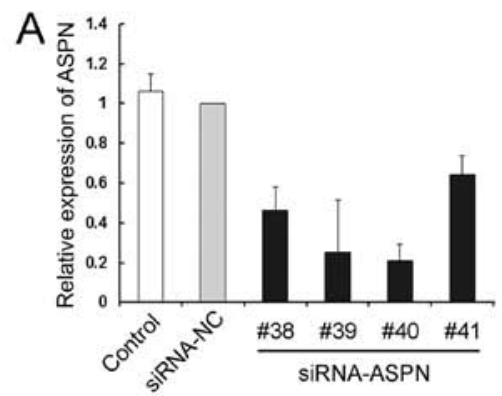

B
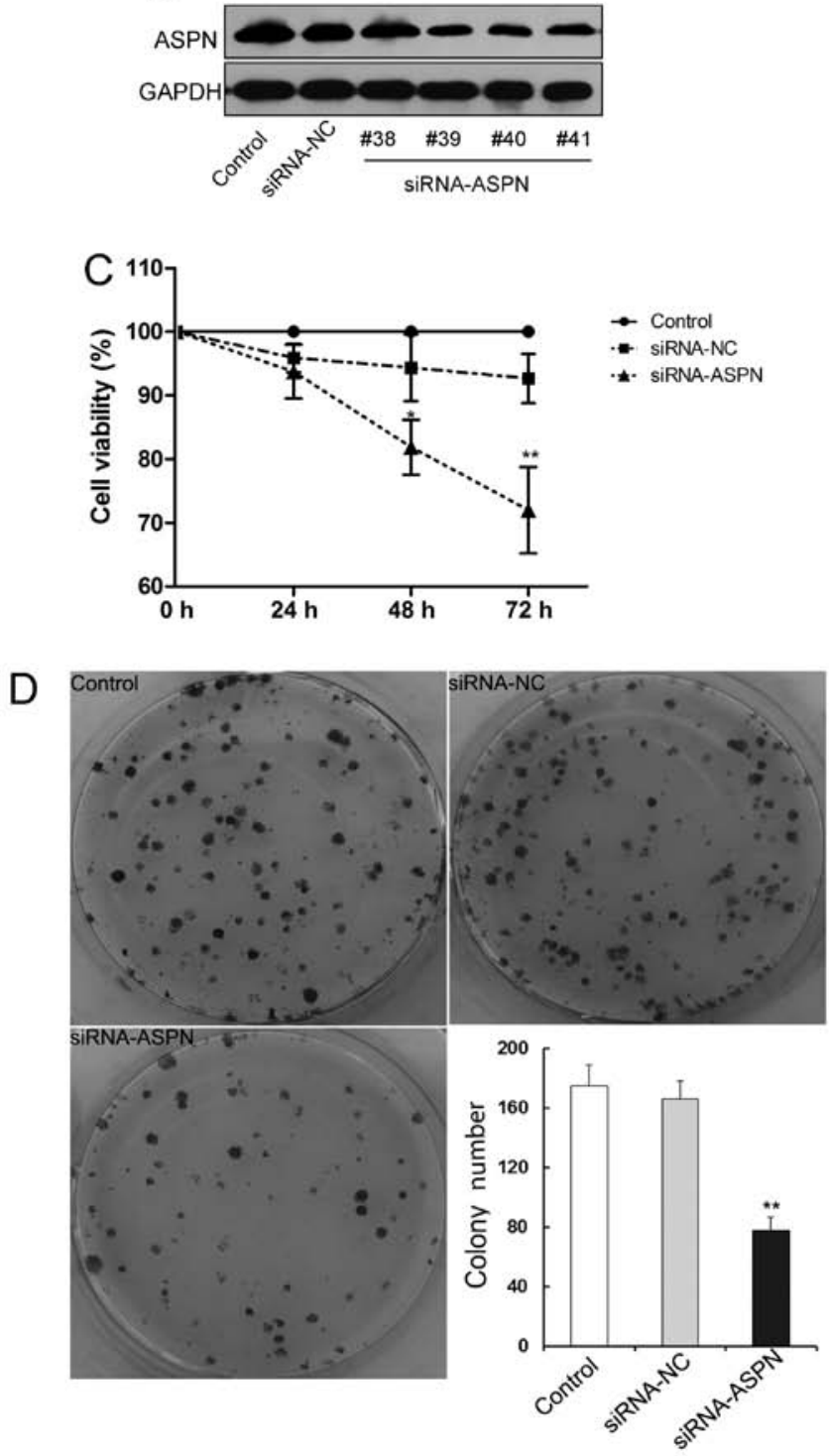

Figure 2. ASPN knockdown decreases the cell viability and clone formation in gastric epithelial cells. (A) Four siRNAs targeting the indicated gene selected and ASPN expression as identified by quantitative real-time PCR. A marked decrease in ASPN mRNA is evident in the siRNA-ASPNGV248 (\#40) transfectants relative to the mock- and control transfectants. (B) ASPN protein levels were assessed by western blotting in AGS cells after siRNA-ASPN-GV248 or siRNA-NC-GV248 vector transfection for $48 \mathrm{~h}$. (C) The cell viability levels were measured by the CCK- 8 assay. The assay was performed at 24,48 or $72 \mathrm{~h}$ in AGS cells transfected with siRNAASPN-GV248 (\#40) or siRNA-NC-GV248 control vector. Experiments were performed in triplicate. (D) The monolayer colony formation assays. AGS cells were transfected with siRNA-ASPN-GV248 (\#40) or siRNA-NCGV248 control vector and selected with G418 for 2 weeks. The downregulated expression of ASPN significantly inhibited the colony formation of AGS cells. Data shown are from representative experiments repeated 3 times with similar results. ${ }^{*} \mathrm{P}<0.05,{ }^{* *} \mathrm{P}<0.01$. ASPN, asporin.

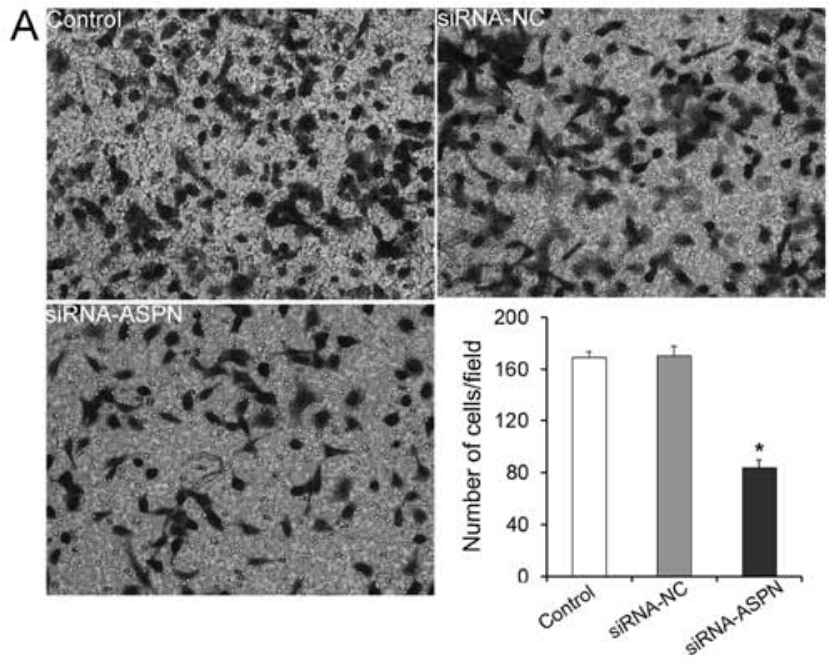

B
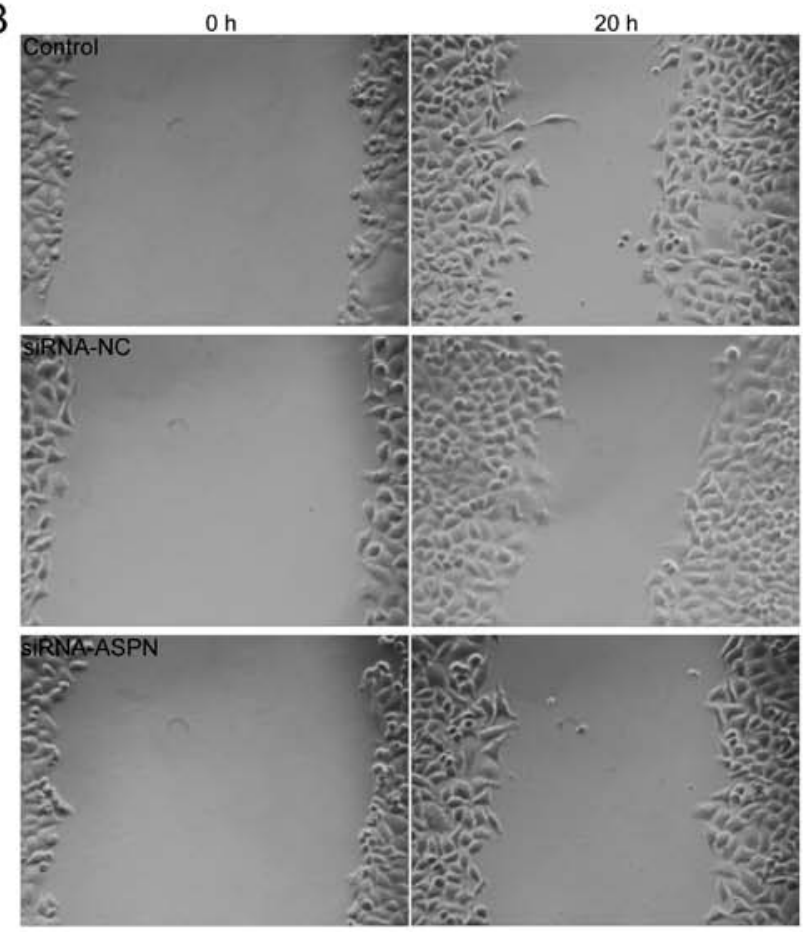

Figure 3. The influence of ASPN knockdown on the migratory ability of gastric cancer cells. (A) The directed migratory capacities of gastric cancer cells after tranfection with the siRNA-ASPN (\#40) were evaluated by a Transwell assay. The cells on the undersurface of the filter were photographed and are shown. Values are the mean \pm SD from 3 independent experiments. ${ }^{*} \mathrm{P}<0.05$. (B) A wound-healing assay was performed to assess the migration of AGS cells after transfection with siRNA-ASPN-GV248 (\#40) or siRNANC-GV248. The migratory rate was quantified by measuring the distance between the edges of the scratch at $\mathrm{t}=0 \mathrm{~h}$ relative to $\mathrm{t}=20 \mathrm{~h}$. ASPN, asporin

transfectants (Fig. 3B). These results indicated that ASPN downregulation reduced the migratory ability of gastric cancer cells. Conversely, it could be concluded that ASPN expression levels were correlated with the gastric cancer migration levels.

ASPN is critical for the activation of the EGFR/ERK signaling pathway. To further substantiate the proliferation and migration activity of ASPN, apoptosis-related proteins (Bcl-2 and Bax) and migration-related proteins (CD44 and MMP-2) were analyzed after silencing of the expression of ASPN. As expected, 

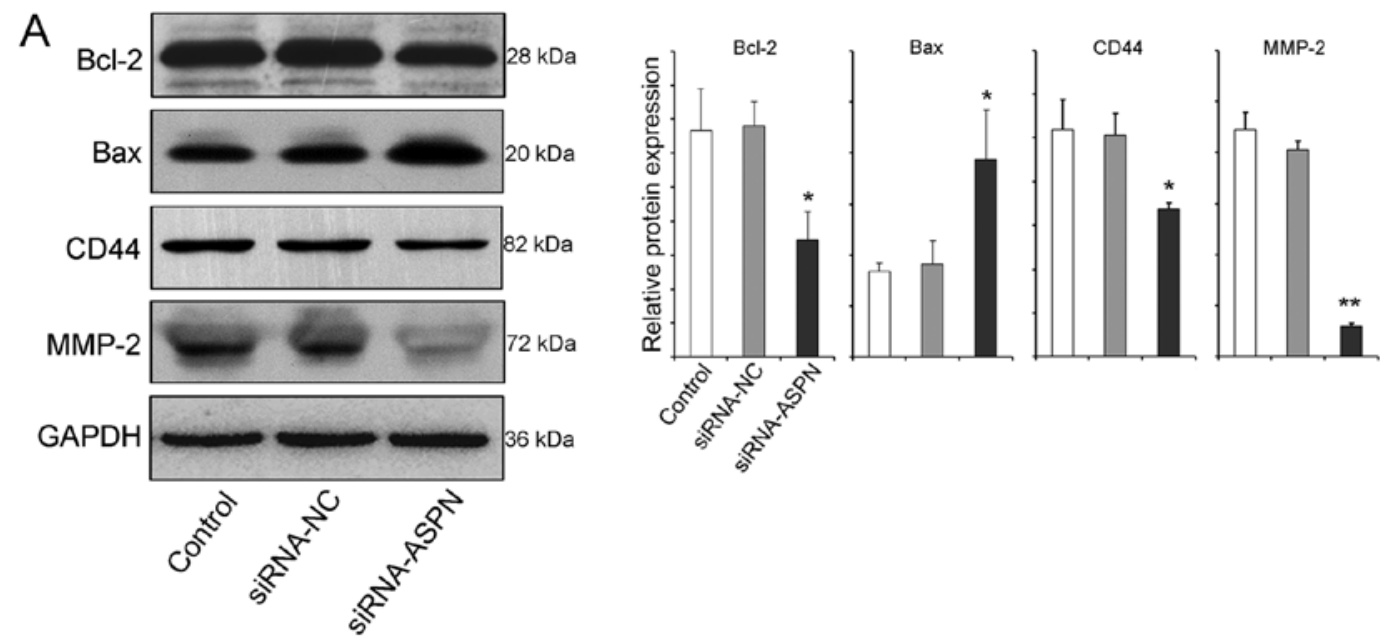

B
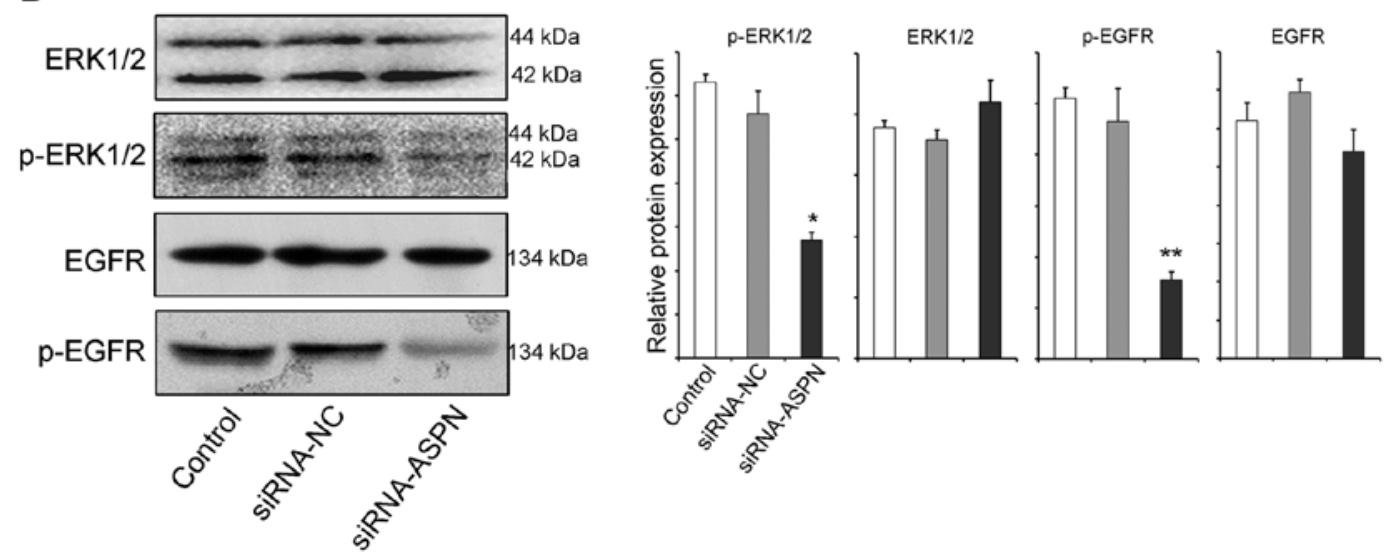

Figure 4. ASPN silencing reduces the activity of the EGFR/ERK pathway. Protein indicators related to Bcl-2, Bax, CD44, MMP-2 (A), and ERK1/2, p-ERK1/2, EGFR, p-EGFR (B) were detected by western blotting after AGS cells were transfected with siRNA-ASPN-GV248 (\#40) or siRNA-NC-GV248 for $72 \mathrm{~h}$. ${ }^{*} \mathrm{P}<0.05,{ }^{* *} \mathrm{P}<0.01$. p- indicates the phosphorylated form. ASPN, asporin; EGFR, epidermal growth factor receptor; MMP, matrix metalloproteinase.

the western blot analysis results revealed that the downregulation of ASPN blocked the expression of the anti-apoptotic molecule Bcl-2 and increased the pro-apoptotic molecule Bad, keeping in line with the results of the cell viability and colony formation assays. Meanwhile, ASPN silencing also reduced the levels of the migration-related proteins CD44 and MMP-2, confirming the results of the Transwell and wound-healing assays, at the protein level (Fig. 4A). The EGFR/ERK pathway, with many interactions occurring, has been well characterized as a key element of a complex signaling network involved in cell survival, migration and angiogenesis $(24,25)$. To evaluate the potential influence of ASPN on EGFR/ERK signaling, total and general phosphorylation of expression of the related molecules were measured. As shown in Fig. 4B, ectopic ASPN significantly influenced the activation of the phosphorylation status of ERK and EGFR, but not the total molecules. Thus, these findings indicate the possibility that ASPN is involved in gastric cancer cell growth and migration via EGFR-ERK signaling, at least in part.

\section{Discussion}

The tumor environment is one major factor that determines the behavior of malignant cells, and the ECM proteoglycans are essential components of the structural entity (23). Thus, we hypothesized that aberrant expression of proteoglycans is a more frequent event and plays many physiological and pathological roles in the development and progression of tumors and merits further investigation. It is clear that SLRPs are biologically active components of ECM (26). ASPN, a new member of the SLRPs, is expressed abundantly in several malignant tumors, suggesting its involvement in the tumorigenesis and progression of cancer (6). Our present results, consistent with previous studies, revealed a significantly elevated ASPN expression in carcinoma tissues relative to the matched adjacent non-tumor tissues (11-14). In addition, ASPN had varied expression in an inverse trend according to the differentiation degree of gastric cancer epithelial cell lines, hinting its oncogenic property, to some extent.

ASPN may have numerous ways to contribute to tumor progression. However, owing to the limited studies to date, the association between stromal ASPN expression and clinicopathological tumor characteristics remains largely uncharacterized. Even though Satoyoshi et al (7) reported that ASPN promotes the coordinated invasion of cancer-associated fibroblasts and gastric cancer both in vitro and in vivo, further investigation is required to clarify both the general role and exact mechanisms of action of ASPN in human neoplasia.

On the basis of the literature, we performed cell proliferation assays to evaluate the potential role of ASPN in gastric 
cancer epithelial cell proliferation. Our findings from both the CCK-8 and colony formation assays showed that ASPN blockade abrogates the proliferation of AGS cells. At the protein level, ASPN silencing also induced a decreased level of anti-apoptotic Bcl-2 and an increased level of pro-apoptotic Bad, indicating that ASPN may play an oncogenic role in gastric tumor cell survival and proliferation.

Furthermore, we performed migration assays to investigate whether ASPN influences the migratory ability of gastric cancer cells. To exclude the possibility that cell proliferation influenced cell migration, migration assays were carried out within $24 \mathrm{~h}$ of siRNA transfection, when siRNA-ASPN did not induce a significant reduction in cell viability. Our results revealed that siRNA-mediated knockdown of ASPN in AGS cells significantly decreased the migration of gastric cancer cells. CD44 together with other oncoproteins, such as MMP-2 and MMP-9, have been reported to be associated with gastric cancer metastasis (27). Khurana et al recently documented that loss of CD44 in vivo results in decreased proliferation of gastric epithelium and the ERK $\rightarrow$ CD44 $\rightarrow$ STAT3 signaling pathway regulates normal and atrophy-induced gastric stem/progenitor-cell proliferation (28). Additionally, lines of evidence suggest that MMPs participate in cell growth, angiogenesis and epithelial-mesenchymal transition (3). Particularly MMP-2, regulated by tissue inhibitor of MMP-2, was proven to play an important role in the progression of gastric cancer (29). Particularly, the activation of the MMP-2 protein is positively related to the metastasis of gastric cancer AGS cells (30). In this context, our data showed that the expression levels of CD44 and MMP-2 were decreased significantly when ASPN was silenced, which further suggests that ASPN is related to the migration of gastric cancer cells.

An ASPN allele with a 14-aspartic acid repeat in the $\mathrm{N}$-terminal region, was designated as D14. The frequency of the D14 allele was found to increase with disease severity, and the effect of ASPN on TGF- $\beta$ activity was allele-specific (15). Song et al also reported that the D14 allele is associated with lumbar disc degeneration (31). Likewise, through its leucine-rich repeat motif, ASPN inhibits the activation of bone morphogenetic protein receptor, resulting in the inactivation of differentiation of periodontal ligament cells (32). Nevertheless, the biological mechanisms of ASPN involved in the carcinogenesis and metastasis of tumors are not yet fully clarified.

Decorin, with a similar structure to ASPN, is a multifunctional molecule of the ECM (33). Previous evidence suggests that decorin, as a key component of the tumor stroma, interacts with various growth factors such as EGF and TGF- $\beta$ and is necessary for cell migration $(34,35)$. Furthermore, it may regulate the action of several tyrosine-kinase receptors, including the EGFR, ERK and insulin-like growth factor receptor I $(36,37)$. Thereupon, whether similar mechanisms of ASPN play a role in tumors warrants attention. In the present study, ectopic ASPN significantly blocked the activation of the phosphorylation status of ERK and EGFR, but not the total molecules.

Because of the causal involvement in gastric cancer differentiation and progression, EGFR and its ligands have recently received considerable attention (17). MAPK/ERK, one classic downstream signal of the EGFR pathway, could regulate $\mathrm{CD} 44$ to modulate tumor aggressiveness (38). The most recent study showed that prostate cancer cell migration and invasion were inhibited by reducing MMP-2/-9 expression via the ROS/ERK pathways (39). Based on this evidence and our present results, it seems reasonable to conclude that ASPN regulates gastric cancer metastasis through activation of EGFR and the ERK-CD44/MMP-2 pathway.

In summary, our study implies that ASPN, a proteoglycan that is aberrant expressed in gastric carcinoma tissues and cell lines, possesses characteristics of an oncogene to a certain extent in gastric carcinogenesis. The cell and molecular biological analyses of ASPN suggest that the proteoglycan contributes to gastric cancer growth and metastasis by regulating the EGFR signaling pathway. Thus, our research provides additional support of previous studies that report that ASPN represents a viable therapeutic regimen with which to treat gastric cancer aimed at manipulating the cancer microenvironment.

\section{References}

1. Hartgrink HH, Jansen EP, van Grieken NC and van de Velde CJ: Gastric cancer. Lancet 374: 477-490, 2009.

2. Zhang Z, Miao L, Xin X, et al: Underexpressed CNDP2 participates in gastric cancer growth inhibition through activating the MAPK signaling pathway. Mol Med 20: 17-28, 2014.

3. Sampieri CL, León-Córdoba K and Remes-Troche JM: Matrix metalloproteinases and their tissue inhibitors in gastric cancer as molecular markers. J Cancer Res Ther 9: 356-363, 2013.

4. Grivennikov SI, Greten FR and Karin M: Immunity, inflammation, and cancer. Cell 140: 883-899, 2010.

5. Canavese G, Candelaresi G, Castellano I and Mano MP: Expression of proteoglycan versican in in situ breast lesions: relations between stromal changes, histotype, and invasion. Pathol Res Pract 207: 97-103, 2011.

6. Lorenzo P, Aspberg A, Onnerfjord P, et al: Identification and characterization of asporin. A novel member of the leucine-rich repeat protein family closely related to decorin and biglycan. J Biol Chem 276: 12201-12211, 2001.

7. Satoyoshi R, Kuriyama S, Aiba N, et al: Asporin activates coordinated invasion of scirrhous gastric cancer and cancer-associated fibroblasts. Oncogene 34: 650-660, 2015.

8. Theocharis AD, Vynios DH, Papageorgakopoulou N, et al: Altered content composition and structure of glycosaminoglycans and proteoglycans in gastric carcinoma. Int $\mathrm{J}$ Biochem Cell Biol 35: 376-390, 2003.

9. Hu L, Duan YT, Li JF, et al: Biglycan enhances gastric cancer invasion by activating FAK signaling pathway. Oncotarget 5: 1885-1896, 2014

10. Wang B, Li GX, Zhang SG, et al: Biglycan expression correlates with aggressiveness and poor prognosis of gastric cancer. Exp Biol Med (Maywood) 236: 1247-1253, 2011.

11. Ansari D, Aronsson L, Sasor A, et al: The role of quantitative mass spectrometry in the discovery of pancreatic cancer biomarkers for translational science. J Transl Med 12: 87, 2014.

12. Turtoi A, Musmeci D, Wang Y, et al: Identification of novel accessible proteins bearing diagnostic and therapeutic potential in human pancreatic ductal adenocarcinoma. J Proteome Res 10: 4302-4313, 2011.

13. Klee EW, Bondar OP, Goodmanson MK, et al: Candidate serum biomarkers for prostate adenocarcinoma identified by mRNA differences in prostate tissue and verified with protein measurements in tissue and blood. Clin Chem 58: 599-609, 2012.

14. Turashvili G, Bouchal J, Baumforth K, et al: Novel markers for differentiation of lobular and ductal invasive breast carcinomas by laser microdissection and microarray analysis. BMC Cancer 7: $55,2007$.

15. Kizawa H, Kou I, Iida A, et al: An aspartic acid repeat polymorphism in asporin inhibits chondrogenesis and increases susceptibility to osteoarthritis. Nat Genet 37: 138-144, 2005.

16. Kopp R, Rothbauer E, Ruge M, et al: Clinical implications of the EGF receptor/ligand system for tumor progression and survival in gastrointestinal carcinomas: evidence for new therapeutic options. Recent Results Cancer Res 162: 115-132, 2003. 
17. Moon WS, Tarnawski AS, Chai J, et al: Reduced expression of epidermal growth factor receptor related protein in gastric cancer. Gut 54: 201-206, 2005.

18. Ashktorab H, Daremipouran M, Wilson M, et al: Transactivation of the EGFR by AP-1 is induced by Helicobacter pylori in gastric cancer. Am J Gastroenterol 102: 2135-2146, 2007.

19. Hwang YP, Yun HJ, Choi JH, et al: Suppression of EGF-induced tumor cell migration and matrix metalloproteinase-9 expression by capsaicin via the inhibition of EGFR-mediated FAK/Akt, PKC/Raf/ERK, p38 MAPK, and AP-1 signaling. Mol Nutr Food Res 55: 594-605, 2011.

20. Murphy KM, Ranganathan V, Farnsworth ML, et al: Bcl-2 inhibits Bax translocation from cytosol to mitochondria during drug-induced apoptosis of human tumor cells. Cell Death Differ 7: 102-111, 2000

21. Wobus M, Rangwala R, Sheyn I, et al: CD44 associates with EGFR and erbB2 in metastasizing mammary carcinoma cells. Appl Immunohistochem Mol Morphol 10: 34-39, 2002.

22. Gurgel DC, Valença-Junior JT, Dornelas CA, et al: Immuno expression of metalloproteinases 2 and 14 and TIMP-2 inhibitor in main types of primary gastric carcinomas and lymph node metastasis. Pathol Oncol Res 21: 73-81, 2015.

23. Zhang Z, Zhang J, Miao L, et al: Interleukin-11 promotes the progress of gastric carcinoma via abnormally expressed versican. Int J Biol Sci 8: 383-393, 2012.

24. Schmidt $M$ and Lichtner RB: EGF receptor targeting in therapy-resistant human tumors. Drug Resist Updat 5: 11-18, 2002.

25. Kolch W: Coordinating ERK/MAPK signalling through scaffolds and inhibitors. Nat Rev Mol Cell Biol 6: 827-837, 2005.

26. Merline R, Schaefer RM and Schaefer L: The matricellular functions of small leucine-rich proteoglycans (SLRPs). J Cell Commun Signal 3: 323-335, 2009.

27. Shi M, Zheng D, Sun L, et al: XB130 promotes proliferation and invasion of gastric cancer cells. J Transl Med 12: 1, 2014.

28. Khurana SS, Riehl TE, Moore BD, et al: The hyaluronic acid receptor CD44 coordinates normal and metaplastic gastric epithelial progenitor cell proliferation. J Biol Chem 288: 16085-16097, 2013.
29. Łukaszewicz-Zając M, Mroczko B, Guzińska-Ustymowicz K, et al: Matrix metalloproteinase 2 (MMP-2) and their tissue inhibitor 2 (TIMP-2) in gastric cancer patients. Adv Med Sci 58: 235-243, 2013.

30. Hwang TL, Changchien TT, Wang CC and Wu CM: Claudin-4 expression in gastric cancer cells enhances the invasion and is associated with the increased level of matrix metalloproteinase-2 and -9 expression. Oncol Lett 8: 1367-1371, 2014.

31. Song YQ, Cheung KM, Ho DW, et al: Association of the asporin D14 allele with lumbar-disc degeneration in Asians. Am J Hum Genet 82: 744-747, 2008.

32. Tomoeda M, Yamada S, Shirai H, et al: PLAP-1/asporin inhibits activation of BMP receptor via its leucine-rich repeat motif. Biochem Biophys Res Commun 371: 191-196, 2008.

33. Cabello-Verrugio $\mathrm{C}$ and Brandan E: A novel modulatory mechanism of transforming growth factor-beta signaling through decorin and LRP-1. J Biol Chem 282: 18842-18850, 2007.

34. Zafiropoulos A and Tzanakakis GN: Decorin-mediated effects in cancer cell biology. Connect Tissue Res 49: 244-248, 2008.

35. Mohan RR, Tovey JC, Gupta R, et al: Decorin biology, expression, function and therapy in the cornea. Curr Mol Med 11: 110-128, 2011.

36. Morcavallo A, Buraschi S, Xu SQ, et al: Decorin differentially modulates the activity of insulin receptor isoform A ligands. Matrix Biol 35: 82-90, 2014.

37. Goldoni S, Iozzo RA, Kay P, et al: A soluble ectodomain of LRIG1 inhibits cancer cell growth by attenuating basal and ligand-dependent EGFR activity. Oncogene 26: 368-381, 2007.

38. Judd NP, Winkler AE, Murillo-Sauca O, et al: ERK1/2 regulation of CD44 modulates oral cancer aggressiveness. Cancer Res 72 : 365-374, 2012

39. Chen Y, Zheng L, Liu J, et al: Shikonin inhibits prostate cancer cells metastasis by reducing matrix metalloproteinase-2/-9 expression via AKT/mTOR and ROS/ERK1/2 pathways. Int Immunopharmacol 21: 447-455, 2014. 